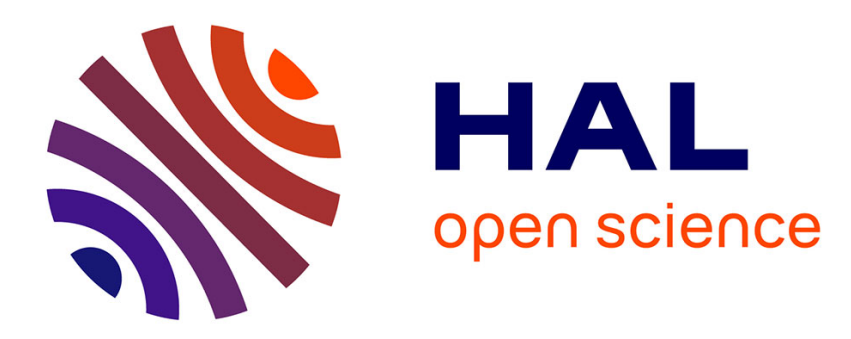

\title{
Quand gauche et droite votent les mêmes lois : Angela Merkel et les réformes Hartz de Gerhard Schröder
}

Pierre Baudry

\section{To cite this version:}

Pierre Baudry. Quand gauche et droite votent les mêmes lois: Angela Merkel et les réformes Hartz de Gerhard Schröder. 2019. hal-02400546

\section{HAL Id: hal-02400546 \\ https://hal.science/hal-02400546}

Preprint submitted on 16 Dec 2019

HAL is a multi-disciplinary open access archive for the deposit and dissemination of scientific research documents, whether they are published or not. The documents may come from teaching and research institutions in France or abroad, or from public or private research centers.
L'archive ouverte pluridisciplinaire HAL, est destinée au dépôt et à la diffusion de documents scientifiques de niveau recherche, publiés ou non, émanant des établissements d'enseignement et de recherche français ou étrangers, des laboratoires publics ou privés. 


\title{
Quand gauche et droite votent les mêmes lois : Angela Merkel et les réformes Hartz de Gerhard Schröder
}

\author{
Pierre Baudry \\ EPHE/CNRS, Paris \\ Université de Tours \\ Tous droits déposés. Pour toute demande, merci de contacter l'auteur à cette adresse : \\ baudrypierre@yahoo.fr
}

Les réformes Hartz constituent un objet privilégié des études sur l'Allemagne. Germanistes, économistes, politistes ont examiné les principes et conséquences des lois Hartz. Mais l'opinion publique et les hommes politiques se sont aussi largement penchés sur ces réformes. Aux yeux de beaucoup, l'Allemagne a en effet engagé une série de transformations majeures de son Etat social et de son marché du travail au travers de ces mesures. L'économie outre-Rhin a semblé en effet s'éloigner du modèle corporatiste qui la caractérise depuis le XIXème siècle. Le modèle rhénan recule-t-il face au modèle libéral anglo-américain ?

Dans ce qui suit, nous voulons nous pencher sur ces questions sous un angle spécifique. Nous voulons examiner ici l'attitude des démocrates-chrétiens dirigés par Angela Merkel face aux réformes Hartz. Ce que nous voulons comprendre, c'est l'attitude de la CDU/CSU en amont et durant le vote des ces lois - Hartz I, II, III et IV. Notre idée centrale consiste en effet à avancer qu'il y a eu collaboration entre la majorité dirigée par Gerhard Schröder et l'opposition démocrate-chrétienne. Les recherches sur l'opposition parlementaire reste de manière relativement négligée dans les études politistes. Ceci est d'autant plus vrai pour les partis démocrates-chrétiens.

Dans le présent article nous voulons contribue à combler cette lacune. Notre hypothèse centrale est la suivante : il y a eu au tournant des années 2000 une mutation symétrique de la $\mathrm{CDU} / \mathrm{CSU}$ et du SPD. Les deux grands mouvements ont connu une évolution idéologique significative vers davantage de libéralisme, une remise en cause de l'Etat-providence et une adaptation marquée aux contraintes de la mondialisation. Il y a donc eu un recentrement évident de la part des grands Volksparteien allemands sous l'impact de la fin de la Guerre froide et du libéralisme anglo-saxon. 
Mais à cette évolution idéologique, il faut ajouter un second élément. La loi fondamentale allemande prévoit en effet que le Bundestag et le Bundesrat trouvent un compromis autour des projets de loi du gouvernement. Mais quand les deux chambres sont dominées par des partis différents comme c'était le cas lors du vote des lois Hartz, alors le SPD et la CDU/CSU doivent trouver un accord. Ce mécanisme lié à la constitution allemande est selon nous le second mécanisme qui explique le rapprochement entre les conservateurs et les sociaux-démocrates au sujet des lois Hartz. Au niveau chronologique, nous nous intéresserons à la période comprise entre 1998 et 2005 qui correspond aux deux mandats de Schröder comme chancelier ${ }^{1}$.

Dans la première partie, nous évoquerons les grandes lignes de la vision économique d'Angela Merkel et les projets de réformes de la CDU/CSU pour les élections législatives fédérales de 2003 et 2005 . Nous étudierons ainsi la prégnance des idées libérales dans le discours des démocrates-chrétiens. Dans la seconde partie nous analyserons les grands axes des lois Hartz pour montrer que ces réformes correspondent à un tournant plus libéral de la part du SPD et des Verts. Dans la troisième partie, nous nous intéresserons au rapprochement idéologique et aux compromis législatifs entre la CDU/CSU et le gouvernement rouge-vert lors du vote des lois Hartz.

\section{Partie I: Après la défaite de 1998, la CDU/CSU accentue l'aspect libéral de son programme}

Le changement de personnel à la tête de la CDU après de Helmut Kohl de la direction du parti démocrate-chrétien en 1998 et les luttes internes sont à mettre en parallèle avec les évolutions idéologiques et programmatiques des partis démocrates-chrétiens durant la phase d'opposition comprise entre 1998 et $2005^{2}$. De manière intéressante, on relèvera que durant

\footnotetext{
${ }^{1}$ Nos sources pour aborder le travail législatif des démocrates-chrétiens nous prendrons en compte les sources suivantes. Nous nous appuyons sur les congrès de la CDU (Parteitage), les procès-verbaux du Bundestag, du Bundesrat sur la période, les rapports des experts auditionnés par la commission parlementaire de l'économie et du travail (Ausschuss für Wirtschaft und Arbeit) entre 1998 et 2005.

${ }^{2}$ Sur les évolutions idéologiques de la CDU, voir Udo Zolleis (2008) Die CDU, Die CDU nach Helmut Kohl: Zwischen Euphorie und Parteikrise-Die Oppositionsjahre 1998 bis 2005.. VS Verlag für Sozialwissenschaften. Simon Green (2013) Societal Transformation and Programmatic Change in the CDU, German Politics, 22:1-2, 46-63, DOI: 10.1080/09644008.2013.795551 Michael Schlieben (2007), Politische Führung in der Opposition, Die CDU nach dem Machtverlust 1998, Innerparteilicher Wettbewerb. In: Politische Führung in der Opposition. VS Verlag für Sozialwissenschaften. Clay Clemens (2009) Modernisation or Disorientation? Policy Change in Merkel's CDU, German Politics, 18:2, 121-139,

Pierre Baudry

EPHE/CNRS, Paris

Université de Tours

Tous droits déposés. Pour toute demande, merci de contacter l'auteur à cette adresse :

baudrypierre@yahoo.fr
} 
cette période la CDU connut deux présidents - Wolfgang Schäuble et Angela Merkel - trois chefs de groupe parlementaire au Bundestag - Schäuble, Merz et Merkel - et deux candidats différents à la chancellerie - Edmund Stoiber en 2002 et Merkel en 2005. Pourtant, la ligne programmatique des partis démocrates-chrétiens ne connut pas de contradictions internes ou ne fut pas marquée par des conflits importants.

Si l'on cherche à éclaircir de manière plus précise les grandes étapes de la redéfinition discursive de la CDU/CSU entre 1998 et 2005, il convient alors de s'intéresser d'abord aux raisons de ce changement idéologique au sein des partis démocrates-chrétiens. Les motivations sont relativement simples à évoquer et sont d'ordre interne et tiennent aux conséquences de la réunification sur l'économie allemande, mais aussi externes liées à l'entrée dans la société «mondialisée » postérieure à 1991. Le thème le plus important au sein du discours des démocrates-chrétiens est l'économie et la «nouvelle économie sociale de marché » (Neue Soziale Marktwirtschaft) qui désigne un ensemble de réformes soutenues par Angela Merkel.

Or, dans le discours de la CDU/CSU, les contraintes liées à la mondialisation, à la mobilité du capital et à l'entrée dans la «société du savoir» (Wissensgesellschaft) liée au développement d'internet sont présentées comme une donnée indépassable. L'enjeu pour l'Allemagne dans le discours des démocrates-chrétiens au tournant des années 2000 porte sur l'adaptation de l'Allemagne à une mondialisation ouverte et hautement compétitive. Nous voulons chercher à déterminer de quelle manière les partis démocrates-chrétiens autour dans les années 1998-2005 ont voulu positionner l'Allemagne dans la mondialisation et le discours qu'ils ont développé durant la phase dans l'opposition. Nous voulons par conséquent comprendre les thèmes et les éléments sémantiques centraux que la CDU/CSU mobilise dans ces discours internes, programmes politiques, mais aussi lors des débats électoraux. Nous nous intéresserons en particulier à quelques signifiants de nature économique et néo-libérale particulièrement importants telle que celui de «modernisation» (Modernisierung), « réforme » (Reform), « réduction de la bureaucratie » (Bürokratieabbau), « mondialisation » (Globalisierung).

Quelles furent les circonstances internes aux partis démocrates-chrétiens allemands qui présidèrent aux évolutions idéologiques de ces partis ? La période d'opposition fut une 
occasion importante pour ce renouvellement discursif initié déjà lors du court mandat de Wolfgang Schäuble comme président fédéral de la $\mathrm{CDU}^{3}$.

Il mit en effet en place dès 1998 des groupes de travail au sein du parti pour contribuer au renouvellement idéologique du parti démocrate-chrétien alors qu'à la différence du SPD par exemple le travail programmatique fut historiquement moins important qu'au sein de la gauche. Ces travaux aboutirent aux «Lignes directrices d'Erfurt» (Erfurter Leitsätze) visant une transformation du discours de la CDU. Ce document entend rompre avec les «tabous » de l'ère Kohl et défend une vision du marché du travail plus centrée sur la flexibilité et les services ${ }^{4}$.

Mais après le départ de Schäuble, c'est Angela Merkel qui prend la tête des démocrates-chrétiens. Le changement majeur introduit par cette dernière à partir de sa prise de pouvoir au sein de la CDU (2000) et du groupe parlementaire de la CDU/CSU (2002) ne tient pas à des innovations idéologiques spécifiques, mais plutôt à son action pour mettre en avant un concept qui lui était cher, celui de «nouvelle économie sociale de marché » (Neue Soziale Marktwirtschaft). Ce concept est d'une très grande importance non seulement en raison de son contenu économico-social, mais aussi des valeurs collectives qu'il mobilise dont le plus important est sans aucun doute celui d' "économie sociale de marché » (soziale Marktwirtschaft).

Penchons-nous d'abord sur les discours internes à la CDU relatifs à la «nouvelle économie de marché ». Son contenu est exposé de manière très générale par Merkel lors du $14^{\text {ème }}$ congrès de la CDU à Dresde en décembre 2001 qui marque un virage idéologique dans la mesure où Angela Merkel y expose la première fois sa vision des réformes économiques nécessaires selon elle. Dans ce discours, Merkel ne propose pas des mesures précises. Elle développe plutôt une vision globale de la voie que doit suivre selon la CDU dans le domaine économique et qui exprime le consensus autour de la politique économique au sein de la CDU/CSU. Elle insiste d'abord sur la nécessité du changement face aux nouveaux défis de la

\footnotetext{
${ }^{3}$ Schlieben.

4 Congrès de la CDU à Erfurt, 25.4.1999 au 27.4.1999. URL : https://www.kas.de/c/document library/get_file?uuid=31db6333-131a-bcc3-19b703a165595d14\&groupId $=252038$

Pierre Baudry

EPHE/CNRS, Paris

Université de Tours

Tous droits déposés. Pour toute demande, merci de contacter l'auteur à cette adresse : baudrypierre@yahoo.fr
} 
mondialisation en raison de l'évolution de l'économie allemande qui fait des années 2000 un tournant dans la vie économique et sociale du pays ${ }^{5}$.

«Chers amis, aujourd'hui, nous sommes confrontés à un nouveau défi. Nous sommes confrontés au défi que les nouveaux développements de la technologie ont changé notre monde. Les technologies de l'information et de la communication ont permis le développement de la mondialisation. Tout s'est accéléré. Aujourd'hui, dans notre ordre démocratique libéral, nous devons parvenir à surmonter des contradictions. Nous devons concilier la contradiction entre la mondialisation d'une part et l'enracinement dans notre propre pays d'autre part. C'est la tâche à laquelle nous, démocrates chrétiens, sommes confrontés en Allemagne 6 . »

Le tournant vers plus de libéralisme de la part de la CDU/CSU ne fait pas de doute si l'on observe les programmes électoraux pour les élections législatives fédérales de 2005 qui verront l'élection de Angela Merkel. Au niveau des mesures politiques, la candidate démocrate-chrétienne proposera une série de réformes majeures au plan économique et qui visent à baisser le niveau de la fiscalité et à libéraliser le marché du travail allemand.

- Merkel entend baisser drastiquement les tranches supérieures de l'impôt sur le revenu. Elle veut simplifier les barèmes en mettant en place uniquement trois tranches $-10 \%$, $20 \%$ et $35 \%$ - et en supprimant toutes les niches fiscales.

- Elle veut supprimer le financement des systèmes de santé par des cotisations salariales et patronales proportionnelles au salaire pour introduire un système de financement forfaitaire et identique pour tous.

- Elle soutiendra aussi une flexibilisation du marché du travail afin de faciliter les licenciements et des mesures pour inciter les chômeurs à reprendre un emploi.

Mais quels sont les points de convergence entre ces propositions et la politique menée par Schröder? Pour répondre à cette question, nous allons examiner l'attitude de la CDU/CSU face aux réformes Hartz.

\footnotetext{
${ }^{5}$ Angela Merkel, $14^{\text {ème }}$ congrès de la CDU à Dresde, du 2-4/12/2001, p. 21.

${ }^{6}$ Angela Merkel, Ibidem. Texte original : «Liebe Freunde, heute stehen wir vor einer neuen Herausforderung. Wir stehen vor der Herausforderung, dass neue technische Entwicklungen unsere Welt verändert haben. Die Informations- und Kommunikationstechnologie hat die Globalisierung vorangebracht. Prozessegehen schneller. Heute müssen wir in unserer freiheitlichen, demokratischen Ordnung es wie-der schaffen, Widersprüche zu versöhnen. Wir müssen den Widerspruch zwischen Globalisierung auf der einen Seite und Verwurzelung in unserem eigenen Land auf der anderen Sei-te versöhnen. Das ist die Aufgabe, vor der wir Christdemokraten in Deutschland stehen. »

Pierre Baudry

EPHE/CNRS, Paris

Université de Tours

Tous droits déposés. Pour toute demande, merci de contacter l'auteur à cette adresse : baudrypierre@yahoo.fr
} 


\section{Partie II : La CDU et les réformes Hartz de Schröder : un consensus libéral entre la droite et la gauche allemande?}

\section{a) Les grandes lignes des réformes Hartz}

Les lois Hartz constituent l'exemple le plus connu et le plus important de la politique de réforme mise en place le gouvernement rouge-vert mené par Schröder. Nous voulons examiner ces lois dans ce qui suit afin de comprendre dans les grandes lignes des mesures prises par les sociaux-démocrates et les Verts, mais aussi afin de déterminer la nature du rapprochement entre les démocrates-chrétiens et la gauche allemande autour des réformes économiques.

Dans ce qui suit, nous entendons ainsi revenir sur la période schröderienne, qui en bien des points constitue selon nous le tournant majeur de l'économie allemande avec l'introduction de l'euro comme monnaie fiduciaire en l'an 2002. Notre intention consiste donc à étudier la réorientation de la politique allemande sous l'impact de l'introduction de l'euro sous forme fiduciaire à partir de l'an 2002 et des nouvelles politiques publiques économiques.

Les grandes lignes des lois Hartz sont les suivantes :

- Les lois Hartz tendent à développer une économie de service en partant du postulat que ces derniers constituent l'avenir dans un contexte historique où que c'est l'industrie qui fait la force économique de l'Allemagne historiquement ${ }^{7}$. Ces lois introduites par le gouvernement Gerhard Schröder veulent de plus engager une stratégie en faveur d'une société du savoir (Wissensgesellschaft) mobilisant la recherche pour développer des secteurs de pointe afin de maintenir l'avance économique et concurrentielle de l'Allemagne. Cette insistance sur l'innovation fait écho notamment avec une des thématiques de la campagne du candidat démocratechrétien, Edmund Stoiber, lors des élections fédérales de 2003.

- Les réformes visent à introduire davantage de «flexibilité » en cherchant à la fois une baisse du coût du travail pour les employeurs en cas de licenciement. Au niveau des services publics, on constate aussi des transformations importantes sous Schröder. Il vise par les lois Hartz à modeler l'agence fédérale de l'emploi (Bundesagentur für Arbeit) sur le modèle d'une entreprise privée. Il veut en faire un prestataire de service

\footnotetext{
7 Werner Abelshauser, Kulturkampf: Der deutsche Weg in die Neue Wirtschaft und die amerikanische Herausforderung. Kulturverlag Kadmos, Berlin 2003.

Pierre Baudry

EPHE/CNRS, Paris

Université de Tours

Tous droits déposés. Pour toute demande, merci de contacter l'auteur à cette adresse :

baudrypierre@yahoo.fr
} 
pour faciliter la reprise d'un emploi du côté des demandeurs d'emploi et la satisfaction des besoins des entreprises en main-d'œuvre ${ }^{8}$.

- De manière générale, Schröder vise aussi une baisse de la fiscalité des foyers et des entreprises au nom d'une logique du niveau d'imposition.

Concernant les réformes Hartz, voici de manière plus détaillée comment Isabelle Bourgeois décrit le contenu de ces quatre lois :

«Entre 2003 et 2005 sont entrées en vigueur quatre lois qui amendent profondément le Code social allemand (Sozialgesetzbuch: SGB). Intitulées «Lois pour des services modernes sur le marché de l'emploi» (Gesetze für moderne Dienstleistungen am Arbeitsmarkt), elles sont d'entrée de jeu surnommées "Lois Hartz ». Elles émanent en effet d'un catalogue de recommandations (...) formulées par une commission ad hoc instituée au début de 2002 (...) : la « Commission pour la réduction du chômage et la restructuration de l'Office fédéral du travail» (Kommission zum Abbau der Arbeitslosigkeit und zur Umstrukturierung der Bundesanstalt für Arbeit). Composée d'experts, de responsables politiques et de partenaires sociaux, elle est présidée par Peter Hartz, auparavant directeur du travail (DRH) chez Volkswagen en sa qualité de membre d'IG Metall' (...).

La première Loi Hartz «(...) Erstes Gesetz für moderne Dienstleistungen am Arbeitsmarkt du 23 décembre 2002, entrée en vigueur le $1^{\text {er }}$ janvier 2003 pour l'essentiel de ses dispositions, pose les premiers jalons visant à fluidifier la rotation chômage/emploi. Elle intervient à deux niveaux principalement : d'une part elle assouplit la réglementation du travail et modernise l'organisation du placement; de l'autre, elle vise à faciliter la reprise d'un emploi par les chômeurs en aménageant la " politique active » pour l'emploi (... $)^{10}$. »

La deuxième loi Hartz « (...) La loi Zweites Gesetz für moderne Dienstleistungen am Arbeitsmarkt du 23 décembre 2002, entrée en vigueur progressivement entre le 01-01 et le

\footnotetext{
$8 \quad$ Source : $\quad$ http://www.sozialpolitik-aktuell.de/tl files/sozialpolitikaktuell/_Politikfelder/Arbeitsmarkt/Dokumente/hartzteill.pdf p. 67 sq.

${ }^{9}$ Isabelle Bourgeois, «Les réformes Hartz, remise en cause de l'Etat social?», Regards sur l'économie allemande, 108 | 2013, 15-35. URL : http://journals.openedition.org/rea/4526.

10 Isabelle Bourgeois, ibidem. Texte de la deuxième loi du 23 décembre 2002 : http://www.bmas.de/SharedDocs/Downloads/DE/PDF-Gesetze/zweites-gesetz-fuer-moderne-dienstleistungenam-arbeitsmarkt-2-gesetz1.pdf? blob=publicationFile. Sur les mini-jobs et la deuxième loi Hartz, voir : Brigitte Lestrade, «Les mini-jobs, une perspective pour les chômeurs? », Regards sur l'économie allemande, 67, juillet 2004. URL : http://journals.openedition.org/rea/3795.

Pierre Baudry

EPHE/CNRS, Paris

Université de Tours

Tous droits déposés. Pour toute demande, merci de contacter l'auteur à cette adresse : baudrypierre@yahoo.fr
} 
01-04-2003, cherche, pour sa part, à rendre plus attractive la reprise d'une activité et à faciliter l'insertion sociale par l'emploi ${ }^{11}$. »

La troisième loi Hartz «(..) Drittes Gesetz für moderne Dienstleistungen am Arbeitsmarkt du 27 décembre 2003 et entrée en vigueur le 01-01-2004 se concentre sur le régime d'assurance chômage. Elle réorganise l'Office fédéral du Travail de Nuremberg, rebaptisé Agence fédérale pour l'Emploi (Bundesagentur für Arbeit). Ses missions sont désormais celles d'un " prestataire de services » de proximité, proche du client ${ }^{12}$. »

La quatrième loi Hartz « (...) Viertes Gesetz für moderne Dienstleistungen am Arbeitsmarkt adoptée le 29 décembre 2003 et entrée en vigueur le 01-01-2005 réorganise entièrement le dispositif d'assistance (...). Jusque là, deux types de régimes existaient, et leurs prestations étaient cumulables: d'une part l'aide aux chômeurs en fin de droits (Arbeitslosenhilfe), d'autre part l'aide sociale (Sozialhilfe) ${ }^{13}$. » Les indemnisations chômage sont désormais remplacées par l'aide sociale au bout d'un an de chômage.

Mais quelle est la genèse de ces lois ? Et quel a été le rôle de la CDU/CSU dans le vote de ces lois?

Or, dans ce contexte il y a sans doute peu de politique et de réformes économicosociales qui ont suscité autant de débats que les réformes dites « Hartz». Cet ensemble législatif décidé par la coalition rouge-verte réunissant le SPD et les Verts (Bündnis 90/Die Grünen) dirigée par Gerhard Schröder représente un tournant dans les deux mandats du chancelier social-démocrate et de la politique économique de l'Allemagne contemporaine.

Nous voulons en effet intégrer dans notre étude l'examen du point de vue des démocrates-chrétiens sur les réformes Hartz. Etant donné que ces réformes ont été portées par le gouvernement rouge-vert de Gerhard Schröder, c'est en effet souvent le point de vue de ce dernier qui a été mis en avant dans l'étude de la « mise à l'ordre du jour » de ces politiques de transformation de l'Etat social et du marché du travail. Or, les discours ou les prises de position des démocrates-chrétiens au tournant des années 2000 par rapport à ces mesures législatives ont été plus rarement étudiées. C'est à cette tâche que nous voulons nous atteler

\footnotetext{
${ }^{11}$ Isabelle Bourgeois, ibidem.

${ }^{12}$ Isabelle Bourgeois, ibidem. Le ministre de l'économie Clement présente en détail la loi Hartz III en septembre 2003, Plenarprotokoll, p. 5736 sqq., URL : http://dip21.bundestag.de/dip21/btp/15/15067.pdf. Projet de loi Hartz III : Plenarprotokoll 15/32, page 2479B - 2493C. URL : http://dip21.bundestag.de/dip21/btp/15/15032.pdf.

${ }^{13}$ Isabelle Bourgeois, Ibidem. Projet de loi Hartz IV : http://dip21.bundestag.de/dip21/btd/15/016/1501638.pdf

Pierre Baudry

EPHE/CNRS, Paris

Université de Tours

Tous droits déposés. Pour toute demande, merci de contacter l'auteur à cette adresse : baudrypierre@yahoo.fr
} 
en nous intéressant non seulement aux procédures de vote, aux débats au Bundestag et le lien entre ces derniers et l'idéologie de la CDU/CSU que nous avons exposée plus haut ${ }^{14}$.

Après un premier mandat qui cherchait à rompre les années Kohl, le gouvernement rouge-vert de Schröder décida au début du deuxième mandat de lancer une série de réformes du travail du marché, de l'Etat social et de la politique du travail allemande. Le discours du 14 mars 2003 du chancelier Schröder devant le Bundestag représente de ce point de vue un tournant dans la politique du dirigeant du $\mathrm{SPD}^{15}$.

Il y évoque d'abord la baisse du coût du travail et en particulier des charges patronales qui mènent à un coût du travail supérieur à celui des autres pays de l'OCDE.

«La situation - tout le monde le ressent ici au sein de cette assemblée, mais aussi à l'extérieur - est extrêmement tendue au niveau international et national. La crise d'Irak pèse lourdement au niveau mondial une conjoncture économique déjà instable. En outre, l'Allemagne doit - et ceci doit aussi être relevé - lutter contre une croissance faible, qui a également des causes structurelles. Les coûts de main-d'œuvre non salariaux ont atteint un niveau qui est devenu un fardeau difficilement supportable pour les travailleurs et qui constitue un obstacle pour les employeurs qui souhaitent créer davantage d'emplois ${ }^{16}$. »

Les évolutions idéologiques chez les sociaux-démocrates sont évidentes. Elles expliquent le rapprochement entre les démocrates-chrétiens et la gauche allemande autour d'une politique plus libérale.

\footnotetext{
${ }^{14}$ La littérature sur les réformes Hartz est considérable en français tout comme en allemand et anglais. On citera notamment: Hüther, Michael; Scharnagel, Benjamin (2005): Die Agenda 2010: Eine wirtschaftspolitische Bilanz.In: APuZ 32-33/2005, S. 23-30. Schmid, Günther (2005): Die Zukunft der Agenda 2010. In: Politische Akademie der Friedrich-Ebert-Stiftung (FES) (Hrsg.): Soziale Demokratie in Europa. Bonn: FES, S. 173-184. Dingeldey, Irene (2010): Agenda 2010: Dualisierung der Arbeitsmarktpolitik. In: APuZ 48/2010, S. 18-25. Heglich, Simon; Knollmann, David; Kuhlmann, Johanna (2011): Agenda 2010. Wiesbaden: VS Verlag.

${ }^{15}$ Regierungserklärung von Bundeskanzler Gerhard Schröder vor dem Deutschen Bundestag am 14. März 2003 in Berlin: "Mut zum Frieden und Mut zur Veränderung”; Bulletin Nr. 21-1 vom 14. März 2003; Herausgeber: Presse- und Informationsamt der Bundesregierung. URL : http://gerhardschroeder.de/2003/03/14/regierungserklarung-agenda-2010/

${ }^{16}$ Ibidem. Texte original : «Die Lage - das spürt jeder hier im Haus, aber auch draußen - ist international wie national äußerst angespannt. Die Krise um den Irak belastet weltweit die ohnehin labile Konjunktur. Deutschland hat darüber hinaus - das gilt es ebenfalls zu sehen - mit einer Wachstumsschwäche zu kämpfen, die auch strukturelle Ursachen hat. Die Lohnnebenkosten haben eine Höhe erreicht, die für die Arbeitnehmer zu einer kaum mehr tragbaren Belastung geworden ist und die auf der Arbeitgeberseite als Hindernis wirkt, mehr Beschäftigung zu schaffen. »

Pierre Baudry

EPHE/CNRS, Paris

Université de Tours

Tous droits déposés. Pour toute demande, merci de contacter l'auteur à cette adresse : baudrypierre@yahoo.fr
} 


\section{b) Le rapprochement idéologique entre les conservateurs et les sociaux-démocrates}

Penchons-nous sur le discours du SPD et des Verts dans les débats du Bundestag pour déterminer dans quelle mesure un langage plus libéral y apparait, mais surtout jusqu'où ce discours rappelle celui de la CDU/CSU dont nous avons souligné plus haut le tournant vers des positions plus libérales à partir de 1998.

Ces réformes législatives furent portées par Wolfgang Clement (SPD) qui fut de 2002 à 2005 ministre fédéral de l'économie et du travail (Bundesminister für Wirtschaft und Arbeit) et membre du conseil d'administration du groupe de réflexion Initiative Neue Soziale Marktwirtschaft (INSM) de 2000 à 2002. Cette association joua un rôle important comme groupe de pression souvent considéré comme assez libéral dans ses orientations politiques et axé sur des réformes du marché du travail et de l'Etat-providence. Peu après avoir quitté la vie politique, Wolfgang Clement devient membre du conseil de surveillance du groupe énergétique RWE et de plusieurs autres grands groupes. On voit par conséquent que le rapprochement entre le monde de l'entreprise et les dirigeants du SPD apparait clairement. Ceci rapprochement est d'autant plus évident entre le SPD et les démocrates-chrétiens que le groupe de réflexion Initiative Neue Soziale Marktwirtschaft (INSM) dont faisait partie Clement fut aussi un des soutiens du projet de Neue Soziale Marktwirtschaft d'Angela Merkel. La INSM est selon certains observateurs un des éléments clés expliquant la percée des idées de réforme libérale en Allemagne au tournant des années $2000^{17}$.

Mais l'influence idéologique du libéralisme apparaît déjà au niveau du discours de la commission Hartz qui reprend le langage de la « réforme » :

«L'économie mondiale traverse actuellement une phase difficile caractérisée par de sérieux processus d'ajustement. La mondialisation, l'intégration européenne et la transition vers une société de la connaissance et des services sont souvent trop perçues comme une menace plutôt qu'une opportunité (...). Une action politique responsable nécessite un examen continu et critique du besoin de réglementations légales et réglementaires ou des obstacles à une croissance plus importante. En outre, il faut rendre les décisions des administrations plus efficaces et plus rapides. La réduction de la bureaucratie doit être encouragée et remplacée par

\footnotetext{
${ }^{17} \mathrm{https}: / /$ www.boeckler.de/pdf/fof_insm_studie_09_2004.pdf

Pierre Baudry EPHE/CNRS, Paris Université de Tours Tous droits déposés. Pour toute demande, merci de contacter l'auteur à cette adresse : baudrypierre@yahoo.fr
} 10 
des services adaptés aux citoyens. Les lois et les réglementations doivent en outre être restraintes à ce qui est nécessaire et supportable ${ }^{18}$. »

Ce langage qui incite à une transformation de l'Etat afin de l'adapter à la mondialisation se retrouve se retrouve aussi dans le programme en 20 points présentés par le Conseil allemand des experts économiques (Sachverständigenrat zur Begutachtung der gesamtwirtschaftlichen Entwicklung) qui fut lui aussi à l'origine des préconisations mises en place par le gouvernement de Gerhard Schröder ${ }^{19}$.

Les débats parlementaires et les travaux législatifs préparatifs en amont du vote des quatre lois Hartz témoignent aussi de la proximité idéologique entre les milieux patronaux et les projets de loi portés par le gouvernement Schröder. Les propos de la confédération fédérale des organisations des employeurs allemands (Bundesvereinigung der Deutschen Arbeitgeberverbände) qui est la plus ancienne de ce type en Allemagne lors d'une audition par la commission parlementaire de l'économie et du travail au Bundestag (Ausschuss für Wirtschaft und Arbeit) sont significatifs du rapprochement idéologique entre les milieux libéraux et le SPD :

«Le marché du travail allemand traverse une mauvaise passe. Plus personne ne conteste ce jugement aujourd'hui. Cependant, les préconisations pour remédier à ces difficultés divergent. L'opinion dominante, partagée par tous les instituts économiques - si l'on met à part ceux proches des syndicats - les organisations économiques internationales et les institutions supranationales, telles que la Commission de l’Union européenne, recommande vivement une déréglementation à grande échelle de la constitution du marché du travail allemand. Celles-ci incluent le droit du travail ainsi que l'assurance chômage. Les deux

18 Bericht Hartz Kommission, p. 38, URL : http://www.sozialpolitik-aktuell.de/tl files/sozialpolitikaktuell/ Politikfelder/Arbeitsmarkt/Dokumente/hartzteil1.pdf. Texte original : « «Die Weltwirtschaft befindet sich gegenwärtig in einer schwierigen Phase mit gravierenden Anpassungsprozessen. Globalisierung, europäische Integration und Übergang zur Wissens- und Dienstleistungsgesellschaft werden häufig zu sehr als Bedrohung statt als Chance erkannt. (...). Verantwortungsbewusstes politisches Handeln erfordert die kontinuierliche und kritische Überprüfung, inwieweit rechtliche Regelungen und Verordnungen notwendig sind oder Hemmnisse für schnelleres Wachstum darstellen. Zudem muss das Verwaltungshandeln effektiviert und beschleunigt werden. Bürokratieabbau muss vorangetrieben und durch bürgerfreundliche Dienstleistungen ersetzt werden. Gesetze und Verordnungen sind dazu in dem erforderlichen wie tragbaren Umfang zu reduzieren. »

${ }^{19}$ Frankfurter Allgemeine Zeitung, https://www.faz.net/aktuell/wirtschaft/sachverstaendigenrat-20-punkte-fuerbeschaeftigung-und-wachstum-181883.html

Pierre Baudry

EPHE/CNRS, Paris

Université de Tours

Tous droits déposés. Pour toute demande, merci de contacter l'auteur à cette adresse : baudrypierre@yahoo.fr 
systèmes portent clairement une «responsabilité » partagée dans la crise de l'emploi en Allemagne ${ }^{20}$. »

\section{c) La coopération entre les conservateurs et les sociaux-démocrates allemands au niveau législatif}

On peut trouver des points de rencontres nombreux entre les idées libérales au sens large du terme et les réformes de Schröder, mais cette continuité idéologique entre le SPD et la CDU/CSU apparaît au sein même du processus législatif qui permit le vote des lois Hartz. La loi fondamentale allemande prévoit en effet que le gouvernement, la chambre basse et la chambre haute partagent l'initiative des lois, mais que tous les projets législatifs soient soumis à la ratification par les deux chambres.

Ce que nous voulons étudier ici sont par conséquent les processus législatifs qui permirent le vote des lois Hartz avec les voix des démocrates-chrétiens.

Pour comprendre cela, il faut se tourner vers les débats parlementaires et rappeler un point important relatif à la procédure législative allemande. Celle-ci prévoit en effet un rôle important à la chambre haute, le Bundesrat, qui représente les Länder et qui est dominée par la CDU/CSU lors du vote des quatre lois Hartz. C'est donc dans ce cadre constitutionnel qu'il faut étudier le rôle des démocrates-chrétiens dans le vote des réformes Hartz. De plus, ces dernières portent clairement la marque d'un tournant libéral dans la politique du SPD. Le rôle de la CDU/CSU doit être analysé à la fois sous l'angle idéologique et proprement législatif.

Or, si on examine la chronologie du vote des réformes Hartz qui occupèrent l'ensemble du second mandat de Gerhard Schröder, on observe que les voix de la CDU/CSU furent indispensables pour obtenir une majorité autour de ces propositions de lois ${ }^{21}$.

- 15 novembre 2002 : le Bundestag (chambre basse) vote les lois Hartz I et II qui contraignent notamment les chômeurs à accepter les offres qui leur sont proposées.

http://webarchiv.bundestag.de/archive/2005/0825/parlament/gremien15/a09/eAnhoerungen/cReformen_am_Arb eitsmarkt/Materialien8 9 2003.pdf. Texte original : «Der deutsche Arbeitsmarkt ist in schlechter Verfassung. Diese Bewertung wird heute von Niemandem mehrbestritten. Allerdings divergieren die vorgeschlagenen Lösungsansätze. Die ganz überwiegende Meinung, getragen von allen - nicht gewerkschaftsnahen Wirtschaftsinstituten, internationalen Wirtschaftsorganisationen und supranationalen Institutionen, wie der Kommis-sion der Europäischen Union, rät dringend zu einer durchgreifenden Deregulierung der deutschen Arbeitsmarktverfassung. Dazu gehören das Arbeitsrecht wie auch die Arbeitslosenversicherung. Beide Systeme tragenein klar erkennbares Maß an „Mitschuld“ an der deutschen Beschäftigungskrise. »

${ }^{21}$ Source de la présente chronologie est le journal TAZ, URL : Source : https://taz.de/!5196513/

Pierre Baudry

EPHE/CNRS, Paris

Université de Tours

Tous droits déposés. Pour toute demande, merci de contacter l'auteur à cette adresse :

baudrypierre@yahoo.fr 
- 20 décembre 2002 : le Bundesrat (chambre haute) contrôlé par les démocrateschrétiens approuve le compromis relatif aux lois Hartz I et II. Ces lois entrent en vigueur le 1er janvier $2003^{22}$.

- 17 octobre 2003 : les lois Hartz III et IV sont voté au Bundestag.

- 19 décembre 2003 : Après de longues négociations au Bundestag et au Bundesrat respectivement contrôlé par le SPD et par la CDU/CSU - Hartz III et IV sont votés.

- 1er janvier 2004 : entrée en vigueur de Hartz III.

- 2 juin 2004 : la mise en place de Hartz IV est retardée pour des questions financières. Un compromis est trouvé entre le SPD et les Verts d'un côté et la CDU/CSU de l'autre côté.

- 1er janvier 2005 : avec l'introduction de Hartz IV, 2,8 millions de personnes perdent leurs droits à l'allocation au chômage et touchent désormais uniquement l'aide sociale. En Allemagne de l'Ouest, le montant de cette aide est de 345 euros par mois, à l'est de 331 euros.

Le mécanisme législatif évoqué dans l'introduction de cet article semble donc confirmé. Le SPD et la CDU/CSU furent contraints de trouver des compromis législatifs au Bundestag et au Bundesrat en raison des dispositions prévues par la loi fondamentale allemande.

D’un point de vue législatif, les conservateurs allemands interviennent ainsi sur les propositions de lois de manière importante et obligent le gouvernement mené par Gerhard Schröder à passer par des commissions parlementaires mixtes (Vermittlungssauschuss) rassemblant des parlementaires des deux chambres dont la chambre haute, le Bundesrat, alors contrôlé par l'opposition démocrate-chrétienne depuis 2001. Cette coopération prévue par la constitution allemande eut lieu malgré l'opposition des démocrates-chrétiens au Bundestag contre des projets encore insuffisants selon elle.

Nous ne retracerons pas en détail sur les débats autour du vote des lois Hartz III et IV qui ont été finalement soutenues par la CDU/CSU. Mais il convient de revenir ici sur les débats conclusifs du 19 décembre 2003 au Bundestag relatif aux lois Hartz III et IV, lois les plus connues et les plus débattues de l'ensemble des lois Hartz. Celles-ci montrent le

\footnotetext{
${ }^{22}$ Pour la vision de la CDU/CSU des réformes Hartz lors des débats parlementaires, voir le discours de Merkel de mars 2003. URL : http://dip21.bundestag.de/dip21/btp/15/15032.pdf p. 2493.

Pierre Baudry

EPHE/CNRS, Paris

Université de Tours

Tous droits déposés. Pour toute demande, merci de contacter l'auteur à cette adresse : baudrypierre@yahoo.fr
} 
rapprochement in fine entre la majorité de Schröder et l'opposition menée par Merkel. Schröder remercie l'opposition pour son soutien lors du vote :

«Gerhard Schröder, chancelier fédéral : Monsieur le Président! Mesdames et Messieurs ! C'est aujourd'hui que nous votons l'Agenda 2010. Je m'en réjouis heureux comme tous l'imaginent (...).

Je suis reconnaissant aux membres de l'opposition et aux ministres-présidents qui ont apporté leur aide, et je tiens à reconnaître qu'ils ont aussi leur part dans le fait que finalement on ne parlera plus à l'étranger (...) de la "German disease », la "maladie allemande » donc. (...) Au contraire, il est reconnu que l'Allemagne bouge, qu'elle accepte les défis et qu'en conséquence, elle est devenue de plus en plus attractive pour les investissements étrangers. (...) De quoi s'agissait-il et de quoi s'agit-il encore? Il s'agit de reconstruire les systèmes de sécurité sociale afin qu'ils puissent continuer à fonctionner à l'avenir dans des conditions économiques radicalement modifiées ${ }^{23}$.

(...) A quoi voulions-nous et voulons-nous arriver? Il s'agit de refonder le système de sécurité sociale afin qu'il puisse continuer à fonctionner à l'avenir dans des conditions économiques radicalement différentes. (...) Ces conditions économiques radicalement différentes ont à voir avec qu'on appelle la mondialisation. En ce qui concerne le système de retraite, ils sont également liés à une pyramide des âges radicalement modifiée dans notre société. Il était donc nécessaire de réajuster la relation entre jeunes et seniors, ce qui

23 Gerhard Schröder, débats au Bundestag, p. 7370, 19 décembre 2003. URL: http://dip21.bundestag.de/doc/btp/15/15084.pdf. « Gerhard Schröder, Bundeskanzler: Herr Präsident! Meine sehr verehrten Damen und Herren! Die Agenda 2010 wird heute beschlossen. Ich bin froh darüber; das wird jeder verstehen.

(...) Ich bin denjenigen in der Opposition und unter den Ministerpräsidenten, die mitgeholfen haben, durchausdafür dankbar und will anerkennen, dass auch sie einen Anteil daran haben, dass es nicht zuletzt auch nach dem Urteil des Auslandes - man sollte sich das einmal genauer anschauen - vorbei sein wird mit dem Gerede über „German disease“, die „deutsche Krankheit“" also.

(...) Vielmehr wird anerkannt, dass Deutschland sich bewegt, die Herausforderungen annimmt und als Folge dessen schon seit etlicher Zeit der Standort Deutschland für Investitionen aus dem Ausland zunehmend attraktiver wird.

(...) Worum ging es und worum geht es immer noch? Es geht um den Umbau der sozialen Sicherungssysteme dergestalt, dass sie auch in Zukunft - unter radikal veränderten wirtschaftlichen Bedingungen - funktionieren können.

(...) Die radikal veränderten ökonomischen Bedingungen haben mit dem zu tun, was sich hinter dem Begriff der Globalisierung verbirgt. Bezogen auf die Alterssicherungssysteme, haben sie auch mit einem radikal veränderten Altersaufbau in unserer Gesellschaft zu tun. Deshalb war es erneut notwendig, das Verhältnis zwischen Jung und Alt, das, was man Pakt der Generationen nennen könnte, neu zu justieren. Wir haben das bei der Rente eingeleitet. Wir sind damit nicht am Ende - ein Beispiel dafür, dass der Reformprozess mit dem Beschluss über die Agenda 2010 nicht zu Ende ist, sondern weitergeführt werden muss. Wir haben bei der Gesundheitsreform gemeinsam etwas zuwege gebracht. (...) Aber wichtig ist doch, dass sich Opposition und Regierung in diesem so schwierigen, so vermachteten Bereich auf Bewegung geeinigt haben. »

Pierre Baudry

EPHE/CNRS, Paris

Université de Tours

Tous droits déposés. Pour toute demande, merci de contacter l'auteur à cette adresse : baudrypierre@yahoo.fr 
pourrait appeler un pacte entre les générations. Nous avons commencé avec les pensions de retraite. Nous ne sommes donc pas arrivés au bout du processus - un exemple que le processus de réforme n'est pas arrivé à son terme avec l'Agenda 2010, mais doit se poursuivre. Nous avons réussi à travailler ensemble sur la réforme de la santé. (...) Mais ce qui est important, c'est que l'opposition et le gouvernement se soient mis d'accord pour agir de concert sur cette questions difficile et dont ils ont hérité. "

Quelques minutes après, Merkel lui répond lors de la même session parlementaire :

«Dr. Angela Merkel (CDU/CSU) : Monsieur le Président! Mesdames et Messieurs! L'Allemagne traverse sa pire crise depuis la Seconde Guerre mondiale. (...) L'Allemagne a une dette et un chômage record. L'Allemagne est à la croisée des chemins et des millions de personnes suivent avec un vif intérêt ce sujet, Monsieur le Chancelier, je vous remercie d'avoir dit très clairement que c'est un jour important. (...) Nous avons réussi à trouver un compromis que le groupe parlementaire CDU/CSU peut accepter ${ }^{24}$. »

Le mécanisme central qui permet la rencontre entre la CDU/CSU et le SPD et les Verts est donc double : rapprochement général des partis vers une politique plus centriste et libérale et nécessité de trouver des compromis au Bundestag et au Bundesrat en raison des dispositions de la constitution.

\section{Conclusion}

Les lois Hartz constituent un symbole ambivalent. Pour les uns, elles expriment le volontarisme politique de Schröder qui sut remettre l'Allemagne sur la voie du redressement économique par une série de réformes courageuses. Aux yeux des autres, elles provoquèrent une précarisation importante sur le marché du travail et une remise en cause de l'Etatprovidence. Dans ce qui précède, nous avons voulu analyser ces lois sous un angle relativement négligé. Nous nous sommes en effet intéressés à l'attitude de la CDU/CSU face à ces lois. Or, nous avons d'abord constaté un élément central. Une fois de retour dans

24 Discours d'Angela Merkel, 19 décembre 2003, URL: p. $7372 . \quad$ URL: http://dip21.bundestag.de/doc/btp/15/15084.pdf. Dr. Angela Merkel (CDU/CSU): Herr Präsident ! Meine Damen und Herren! Deutsch-land befindet sich in der schwersten Krise seit dem Zweiten Weltkrieg. (...) Deutschland hat eine Rekordverschuldung und eine Rekordarbeitslosigkeit. Deutschland steht am Scheideweg. Das, was wir heute $\mathrm{zu}$ beraten haben, wird von Millionenvon Menschen mit großem Interesse verfolgt. Herr Bundeskanzler, ich danke dafür, dass Sie sehrdeutlich gesagt haben, dass dies ein wichtiger Tag ist. (...) Es ist uns gelungen, einen Kompromiss zu finden, dem die CDU/CSU-Bundestagsfraktion zustimmen kann.

Pierre Baudry

EPHE/CNRS, Paris

Université de Tours

Tous droits déposés. Pour toute demande, merci de contacter l'auteur à cette adresse : baudrypierre@yahoo.fr 
l'opposition en 1998, les démocrates-chrétiens infléchissent leur discours et insistent davantage sur les réformes de l'Etat-providence, la libéralisation de l'économie et la baisse de la fiscalité. Merkel ont eu un rôle prééminent dans cette évolution.

Mais au niveau idéologique et législatif, nous avons repéré deux phénomènes expliquant le rôle de la CDU/CSU dans le vote des lois Hartz. D'abord, au niveau des discours on a constaté un rapprochement entre les mesures proposées par les démocrateschrétiens et celui des sociaux-démocrates. Cette proximité est visible au plan des programmes électoraux, des débats parlementaires et des textes de loi. De plus, nous avons constaté que la majorité de gauche et l'opposition de droite allemandes ont été amenées à trouver des compromis législatifs aux deux chambres pour permettre de voter les réformes Hartz. En cela, ces mesures ont marqué une transformation importante de l'économie allemande, mais dans un contexte de compromis transpartisan relativement courant outre-Rhin. 